\title{
Blueberry containing diets protect from bone loss induced by sex steroid deficiency differently depending on sex and by mechanisms independent of canonical sex steroid receptor signaling
}

\author{
Johnson OR, ${ }^{1}$ Sato AY, ${ }^{1}$ Pellegrini GG, ${ }^{1,2,3}$ Cregor $M,{ }^{1}$ McAndrews $\mathrm{K},{ }^{1}$ Atkinson \\ $\mathrm{E},{ }^{1}$ Choi RB, ${ }^{1}$ Maiz M, ${ }^{4}$ McCabe LD,${ }^{5}$ McCabe GP,${ }^{5}$ Peacock M, ${ }^{6}$ Weaver $\mathrm{CM},{ }^{4}$ \\ Burr D, ${ }^{1,7}$ Bellido T. ${ }^{1,6,8}$

\begin{abstract}
${ }^{1}$ Department of Anatomy \& Cell Biology, Indiana University School of Medicine, Indianapolis, IN, ${ }^{2}$ CONICET-Universidad de Buenos Aires. Instituto de Inmunología, Genética y Metabolismo (INIGEM). Facultad de Farmacia y Bioquímica-Hospital de Clínicas "José de San Martín”, Buenos Aires, Argentina. ${ }^{3}$ Universidad de Buenos Aires, Facultad de Odontología. Cátedra de Bioquímica Gral y Bucal, Buenos Aires, Argentina. ${ }^{4}$ Department of Nutrition Science, Purdue University, West Lafayette, IN, ${ }^{5}$ Department of Statistics, Purdue University, West Lafayette, IN, ${ }^{6}$ Department of Medicine, Division of Endocrinology, Indiana University School of Medicine, Indianapolis, IN,

${ }^{7}$ Department of Biomedical Engineering, Indiana University School of Medicine, Indianapolis, IN, ${ }^{8}$ Roudebush Veterans Administration Medical Center, Indianapolis, IN.
\end{abstract}

\section{Background and Hypothesis:}

There is an unmet need for interventions that prevent bone loss induced by sex steroid deficiency. Loss of estrogens or androgens increases ROS in bone. We hypothesized that diets containing blueberries with antioxidant properties would protect from bone loss induced by sex steroid deficiency, depending on sex and on the expression of $\mathrm{Nrf2}$, a transcription factor that regulates the endogenous antioxidant response.

\section{Experimental Design:}

SHAM, ovariectomized (OVX), or orchidectomized (ORX) 4-month-old, wild type (WT) or Nrf2 knockout (KO) male and female mice were fed with control diet or diets containing 3 types of blueberries. Bone mineral density (BMD) and bone microarchitecture were measured by dual-energy $\mathrm{x}$-ray absorptiometry and microscopic computed tomography, respectively; and gene expression was quantified in bone RNA by quantitative PCR.

\section{Results:}

Control-fed OVX or ORX mice exhibited the expected BMD loss, to a similar extent, in WT and KO mice. One of the blueberry diets (Montgomery) prevented bone loss, totally in females and partially in males; and prevented $\sim 50 \%$ of microarchitecture deterioration in ORX mice, independently of Nrf2 expression. OVX and ORX decreased expression of estrogen-response-element gene $\mathrm{C} 3$ or androgen-response-element gene Rhox5, respectively, in both WT and KO mice fed control or Montgomery diets, indicating that bone protection is not due to estrogenic/androgenic actions of the diet.

\section{Conclusion and Potential Impact:}

Montgomery diet prevented bone loss due to sex steroid deficiency fully in females and partially in males. Thus, optimal skeletal benefits might be achieved by tailoring antioxidant-rich diets to patients of either sex. 